\title{
On the Existence of Limit Admissible Equilibria in Discontinuous Games*
}

\author{
Guilherme Carmona $^{\dagger}$ \\ University of Surrey
}

December 13, 2018

\begin{abstract}
We consider the existence of limit admissible equilibria, i.e. Nash equilibria in which each player assigns zero probability to the interior of the set of his weakly dominated strategies, in (possibly) discontinuous games. We show that standard sufficient conditions for the existence of Nash equilibrium, such as better-reply security, fail to imply the existence of limit admissible equilibria. We then modify better-reply security to obtain a new condition, admissible security, and show that admissible security is sufficient for the existence of limit admissible equilibria. This result implies the existence of limit admissible equilibria in a Bertrand-Edgeworth competition setting with convex costs analogous to that of Maskin (1986).
\end{abstract}

${ }^{*}$ I wish to thank Atsushi Kajii, two anonymous referees and seminar participants at the SAET conference (Faro 2017) for very helpful comments. Any remaining errors are, of course, my own.

${ }^{\dagger}$ Address: University of Surrey, School of Economics, Guildford, GU2 7XH, UK; email: g.carmona@surrey.ac.uk. 


\section{Introduction}

The motivation of the refinement literature (see van Damme (1991) for a survey) is that the requirements of Nash equilibrium need to be strengthen to obtain a more appealing solution concept in normal-form games. Along this lines, and following Kohlberg and Mertens (1986), it would be desirable to have a solution concept whose existence is generally guaranteed and in undominated strategies.

For games with finite action spaces, Selten's (1975) perfect equilibrium is a way of obtaining the above properties. However, in games with infinite action spaces, several difficulties arise. First, even when players' payoff functions are continuous, it is possible that all Nash (and, therefore, all perfect) equilibria are in weakly dominated strategies (see Simon and Stinchcombe (1995, Example 2.1)). For this reason, one can only hope to obtain general existence results for limit admissible equilibria, i.e. of Nash equilibrium in which each player assigns zero probability to the interior of the set of his weakly dominated strategies.

Furthermore, when some player's payoff function is discontinuous, existence of perfect equilibria requires conditions considerably stronger than the standard ones guaranteeing existence of Nash equilibrium (such as Reny's (1999) better-reply security). Moreover, it is not always the case that perfect equilibria are limit admissible (see Carbonell-Nicolau (2011b)). Other solution concepts have been proposed such as Andersson, Argenton, and Weibull's (2014) robust equilibrium to strategic uncertainty and Bich's (2016) prudent equilibrium, but again their existence and limit admissibility have only been established under assumptions stronger than the standard ones.

In this paper we directly address the existence of limit admissible equilibria. We first show that a condition different from better-reply security is needed. Indeed, we present two examples of better-reply secure games that fail to have limit admissible equilibria: One of these examples fails to have limit admissible strategies and the other, while having limit admissible strategies, fails to have one that is also a Nash equilibrium. 
We then introduce the notion of admissible security and use it to establish the existence of limit admissible equilibrium in mixed strategies. ${ }^{1}$ In contrast with the complicated task of establishing the existence of perfect equilibrium (and, thus, of establishing indirectly and under some additional assumptions the existence of limit admissible equilibria), we show that the task of establishing directly that a limit admissible equilibrium exists is a relatively simple one. Indeed, besides having to assume that limit admissible strategies exist, all it takes is that better-reply security be "appropriately relativized" to the set of limit admissible strategies. In this sense, admissible security is the natural modification of better-reply security to address the existence of limit admissible equilibria.

It is important to note that admissible security is neither stronger nor weaker than better-reply security. It is stronger than assuming that the game obtained by restricting players to choose limit admissible strategies is better-reply secure; this latter condition is, as we show, not sufficient for the existence of limit admissible equilibria. An additional advantage of our approach as compared to analysing the game restricted to limit admissible strategies is that the former does not require a complete characterization of limit admissible strategies; the general auction setting of Jackson and Swinkels (2005) provides an example where this is useful.

The paper is organized as follows. Section 2 presents our notation and general definitions. Some motivating examples, including those showing that better-reply security is not sufficient for the existence of limit admissible equilibrium are in Section 3. Our new condition, admissible security, is introduced in Section 4 and used to establish the existence of limit admissible equilibrium.

Several remarks in Section 4 complement this result, by showing that: (1) More general existence results can be obtained by relativizing Reny's (2016) correspondence security and finite deviation property; (2) an extension of our result for pure strategies is possible in own-strategy quasi-concave games when the set of limit admissible pure strategies to be convex; (3) the relativization of Reny's (1999) payoff security and of

\footnotetext{
${ }^{1}$ Admissible security also yields the existence of pure-strategy limit admissible equilibria when the set of limit admissible pure strategies is convex for each player.
} 
Bagh and Jofre's (2006) weak reciprocal upper semicontinuity together provide sufficient conditions for admissible security; (4) better-reply secure games are admissible secure provided that each non-limit admissible strategy be weakly dominated by a limit admissible strategy (a weaker version of this property suffices).

In Section 4 we relate our existence result with those that follow from Simon and Stinchcombe (1995) for continuous games and from Carbonell-Nicolau (2011b) for discontinuous games; we also briefly compare our approach with that of Harris, Stinchcombe, and Zame (2005). Our results are applied in Section 5 to a BertrandEdgeworth duopoly game with strictly convex costs, modelled along the lines of Maskin (1986). In addition to being an interesting game in its own right, this game is useful to illustrate our results because it is not difficult to characterize its set of weakly dominated strategies and, once such characterization is obtained, applying our results is a relatively simple task.

\section{Notation and Definitions}

Consider a normal-form game $G=\left(X_{i}, u_{i}\right)_{i \in N}$ consisting of a finite set of players $N=\{1, \ldots, n\}$, and, for each player $i \in N$, a compact metric pure strategy set $X_{i}$ and a bounded and measurable real-valued payoff function $u_{i}$ on $X=\prod_{i=1}^{n} X_{i}$.

For all $i \in N, X_{i}$ is endowed with its Borel $\sigma$-algebra. The set of mixed strategies for player $i$ is the set of (Borel) probability measures $M_{i}$ on $X_{i}$, endowed with the narrow topology. For each $x_{i} \in X_{i}$, we write $\delta_{x_{i}}$ for the Dirac measure at $x_{i}$. Let $M=$ $\prod_{i=1}^{n} M_{i}$. Given a profile $\sigma \in M$ of mixed strategies, we write $\tau_{\sigma}$ for the corresponding product measure on $X$. Define, for all $i \in N$ and $\sigma \in M, u_{i}(\sigma)=\int_{X} u_{i}(x) \mathrm{d} \tau_{\sigma}(x)$.

Throughout the paper, the product of any number of sets is endowed with the product topology. Given a player $i \in N$, the symbol $-i$ denotes "all players but $i$ ". Also, $X_{-i}=\prod_{j \neq i} X_{j}$.

A pure strategy $x_{i} \in X_{i}$ is weakly dominated for $i \in N$ if there exists a mixed strategy $\mu_{i} \in M_{i}$ such that $u_{i}\left(x_{i}, x_{-i}\right) \leq u_{i}\left(\mu_{i}, x_{-i}\right)$ for all $x_{-i} \in X_{-i}$ and $u_{i}\left(x_{i}, x_{-i}^{\prime}\right)<$ $u_{i}\left(\mu_{i}, x_{-i}^{\prime}\right)$ for some $x_{-i}^{\prime} \in X_{-i}$. Let $O_{i}$ be the interior of the set of weakly dominated 
strategies for $i$ and $C_{i}=X_{i} \backslash O_{i}$ be its complement.

We say that $\sigma \in M$ is a Nash equilibrium of $G$ if $u_{i}(\sigma) \geq u_{i}\left(\sigma_{i}^{\prime}, \sigma_{-i}\right)$ for each $i \in N$ and $\sigma_{i}^{\prime} \in M_{i}$; the set of Nash equilibria of $G$ is denoted by $E(G)$. A mixed strategy profile $\sigma \in M$ is limit admissible if $\sigma_{i}\left(O_{i}\right)=0$ for each $i \in N$. Let $L(G)$ denote the set of Nash equilibria of $G$ that are limit admissible, its elements being referred to as limit admissible equilibria.

The existence of Nash equilibria is guaranteed under better-reply security, a notion introduced in Reny (1999) and which we now recall; we shall see that better-reply security is not sufficient for the existence of limit admissible equilibria. A game $G=\left(X_{i}, u_{i}\right)_{i \in N}$ is better-reply secure in mixed strategies if, whenever $\left(\sigma^{*}, u^{*}\right) \in$ $\operatorname{cl}(\operatorname{graph}(u))$ and $\sigma^{*}$ is not a Nash equilibrium of $G$, there exist $i \in N, \mu_{i} \in M_{i}$, an open neighborhood $U$ of $\sigma^{*}$ and a real number $\alpha_{i}>u_{i}^{*}$ such that $u_{i}\left(\mu_{i}, \sigma_{-i}\right) \geq \alpha_{i}$ for each $\sigma \in U$.

\section{Motivating examples}

We illustrate with two simple examples that best-reply secure games may fail to have a limit admissible equilibrium. The second example is also an example of a game whose restriction to its set of limit admissible strategies is continuous but, nevertheless fails to have a limit admissible equilibrium.

Example 1. Let $G=\left(X_{i}, u_{i}\right)_{i \in N}$ be a 2-player game on the unit square (i.e. $N=$ $\{1,2\}$ and $\left.X_{1}=X_{2}=[0,1]\right)$ with the following payoff functions: For each $x \in X$,

$$
u_{1}(x)= \begin{cases}1 & \text { if } x_{2} \leq x_{1}<1 \\ 1 & \text { if } x_{1}=1 \text { and } x_{2}=0 \\ 0 & \text { otherwise }\end{cases}
$$

and

$$
u_{2}(x)= \begin{cases}1 & \text { if } x_{2}=0 \\ 0 & \text { otherwise }\end{cases}
$$


It is easy to see that the set of Nash equilibria of $G$ is $\left\{\left(\sigma_{1}, \delta_{0}\right): \sigma_{1} \in M_{1}\right\}$.

We now show that $G$ is better-reply secure in mixed strategies. Let $\left(\sigma^{*}, u^{*}\right) \in$ $\operatorname{cl}(\operatorname{graph}(u))$ be such that $\sigma^{*}$ is not a Nash equilibrium of $G$. Then $\sigma_{2}^{*} \neq \delta_{0}$, i.e. $\sigma_{2}^{*}(\{0\})<1$. Letting $\left\{\sigma_{k}\right\}_{k=1}^{\infty} \subseteq M$ be such that $\left(\sigma_{k}, u\left(\sigma_{k}\right)\right) \rightarrow\left(\sigma^{*}, u^{*}\right)$ and using Portmanteau theorem (e.g. Billingsley (1999, Theorem 2.1, p. 16)), we obtain that

$$
u_{2}^{*}=\lim _{k} u_{2}\left(\sigma_{k}\right)=\lim _{k} \sigma_{k, 2}(\{0\}) \leq \sigma_{2}^{*}(\{0\})<1 .
$$

Since $u_{2}\left(\sigma_{1}, \delta_{0}\right)=1$ for each $\sigma_{1} \in M_{1}$, it follows that the condition in the definition of better-reply security is satisfied with $i=2, \mu_{2}=\delta_{0}, U=M$ and $\alpha_{2}=1$.

Despite being better-reply secure, $G$ has no limit admissible equilibrium since $C_{1}=\emptyset$. Indeed, each $x_{1}<1$ is weakly dominated for $i=1$ by $x_{1}^{\prime} \in\left(x_{1}, 1\right)$ and $x_{1}=1$ is weakly dominated for $i=1$ by $x_{1}^{\prime}=1 / 2$.

The previous example shows that better-reply security is not sufficient for the non-emptiness of $C_{i}$ for each $i \in N$. The following example shows that better-reply secure games with $C_{i} \neq \emptyset$ for each $i \in N$ may fail to have limit admissible equilibria.

Example 2. Let $G=\left(X_{i}, u_{i}\right)_{i \in N}$ be as in Example 1 except that now

$$
u_{1}(1 / 2,1 / 2)=2 \text { and } u_{1}\left(x_{1}, 0\right)=0 \text { for each } 1 / 4<x_{1}<3 / 4 \text {. }
$$

It is clear that $C_{2}=\{0\}$ and that $C_{1}=\{1 / 2\}$; for the latter, each $x_{1}<1$ different than $1 / 2$ is weakly dominated for $i=1$ by $x_{1}^{\prime} \in\left(\max \left\{x_{1}, 3 / 4\right\}, 1\right)$ and $x_{1}=1$ is weakly dominated for $i=1$ by $x_{1}^{\prime}=3 / 4$. It is also easy to see that the set of Nash equilibria of $G$ is $\left\{\left(\sigma_{1}, \delta_{0}\right): \sigma_{1}((1 / 4,3 / 4))=0\right\}$.

We now show that $G$ is better-reply secure in mixed strategies. Let $\left(\sigma^{*}, u^{*}\right) \in$ $\operatorname{cl}(\operatorname{graph}(u))$ be such that $\sigma^{*}$ is not a Nash equilibrium of $G$. Arguing as in Example 1 , we may assume that $\sigma_{2}^{*}=\delta_{0}$ and $u_{2}^{*}=1$. Hence, $\sigma_{1}^{*}((1 / 4,3 / 4))>0$. Letting $\left\{\sigma_{k}\right\}_{k=1}^{\infty} \subseteq M$ be such that $\left(\sigma_{k}, u\left(\sigma_{k}\right)\right) \rightarrow\left(\sigma^{*}, u^{*}\right)$, we have that $\lim _{k} \sigma_{k, 2}(\{0\})=$ $\lim _{k} u_{2}\left(\sigma_{k}\right)=u_{2}^{*}=1$. Thus, by the Portmanteau theorem (e.g. Billingsley (1999, Theorem 2.1, p. 16)) and by $\int_{X_{1}} u_{1}\left(x_{1}, x_{2}\right) \mathrm{d} \sigma_{k, 1}\left(x_{1}\right) \leq 2$ for each $x_{2}>0$ and $k \in \mathbb{N}$, 
we obtain that

$$
\begin{aligned}
& u_{1}^{*}=\lim _{k} u_{1}\left(\sigma_{k}\right) \leq \varlimsup_{k}\left(\sigma_{k, 2}(\{0\}) \sigma_{k, 1}\left(X_{1} \backslash(1 / 4,3 / 4)\right)+2 \sigma_{k, 2}((0,1])\right) \\
& =\varlimsup_{k} \sigma_{k, 1}\left(X_{1} \backslash(1 / 4,3 / 4)\right) \leq \sigma_{1}^{*}\left(X_{1} \backslash(1 / 4,3 / 4)\right)<1
\end{aligned}
$$

Let $\alpha_{1} \in\left(u_{1}^{*}, 1\right)$ and $O_{2}$ be an open neighborhood of $\delta_{0}$ such that $\int_{X_{2}} 1_{[0,3 / 4)} \mathrm{d} \sigma_{2}>$ $\int_{X_{2}} 1_{[0,3 / 4)} \mathrm{d} \delta_{0}-\left(1-\alpha_{1}\right)$ for each $\sigma_{2} \in O_{2} \cdot{ }^{2}$ Let $i=1, \mu_{1}=\delta_{3 / 4}$ and $U=M_{1} \times O_{2}$; since, for each $\sigma \in U, u_{1}\left(\mu_{1}, \sigma_{2}\right)=\sigma_{2}([0,3 / 4]) \geq \int_{X_{2}} 1_{[0,3 / 4)} \mathrm{d} \sigma_{2}>\int_{X_{2}} 1_{[0,3 / 4)} \mathrm{d} \delta_{0}-\left(1-\alpha_{1}\right)=$ $\alpha_{1}>u_{1}^{*}$, it follows that $G$ is better-reply secure.

Recall that $C_{i}$ is a singleton for each player $i$. This implies that the set of limit admissible strategies of each player is also a singleton; hence, the game obtained by restricting players to choose limit admissible strategies is (trivially) continuous.

However, many discontinuous games have limit admissible equilibria. An easy example is provided by a symmetric Bertrand duopoly with constant marginal costs.

Example 3. Suppose that each firm has a constant marginal cost $0<c<1$ and there is one commodity whose demand is $d(x)=1-x$, where $x$ is the lowest price in the market. Each of the two firms sets a price in the unit interval and gets half of the demand when these prices are equal. Thus, this situation can be described by a game $G=\left(X_{i}, u_{i}\right)_{i \in N}$ with $N=\{1,2\}, X_{1}=X_{2}=[0,1]$ and, for each $x \in X$,

$$
u(x)= \begin{cases}\left(\left(x_{1}-c\right)\left(1-x_{1}\right), 0\right) & \text { if } x_{1}<x_{2}, \\ \left(0,\left(x_{2}-c\right)\left(1-x_{2}\right)\right) & \text { if } x_{1}>x_{2} \\ \left(\left(x_{1}-c\right)\left(1-x_{1}\right) / 2,\left(x_{1}-c\right)\left(1-x_{1}\right) / 2\right) & \text { if } x_{1}=x_{2} .\end{cases}
$$

It is easy to check that $C_{i}=[c,(1+c) / 2]$ for each $i \in N$ and that $\left(\delta_{c}, \delta_{c}\right)$ is a Nash equilibrium of $G$ and, thus, a limit admissible equilibrium of $G$. As we shown in the next section, the existence of a limit admissible equilibrium of $G$ also follows from our existence result.

\footnotetext{
${ }^{2}$ The function $1_{[0,3 / 4)}$ is defined by $1_{[0,3 / 4)}\left(x_{2}\right)=1$ if $x_{2} \in[0,3 / 4)$ and $1_{[0,3 / 4)}\left(x_{2}\right)=0$ if $x_{2} \notin$ $[0,3 / 4)$; it is lower semi-continuous. Thus, the existence of $\mathrm{O}_{2}$ follows by Aliprantis and Border (2006, Theorem 15.5, p. 511).
} 


\section{Existence of limit admissible equilibria}

We obtain the existence of limit admissible equilibria by relativizing the notion of better-reply security as follows. For each $i \in N$, let $M_{i}^{0}=\left\{\mu_{i} \in M_{i}: \mu_{i}\left(O_{i}\right)=0\right\}$ and let $M^{0}=\prod_{i \in N} M_{i}^{0}$. We say that a game $G=\left(X_{i}, u_{i}\right)_{i \in N}$ is admissibly secure if

(a) $C_{i} \neq \emptyset$ for each $i \in N$ and

(b) If $\left(\sigma^{*}, u^{*}\right) \in \operatorname{cl}\left(\left\{(\sigma, u(\sigma)): \sigma \in M^{0}\right\}\right)$ and $\sigma^{*}$ is not a Nash equilibrium of $G$, then there exist $i \in N, \mu_{i} \in M_{i}^{0}$, an open neighborhood $U$ of $\sigma^{*}$ and a real number $\alpha_{i}>u_{i}^{*}$ such that $u_{i}\left(\mu_{i}, \sigma_{-i}\right) \geq \alpha_{i}$ for each $\sigma \in U \cap M^{0}$.

Analogously to Reny's (1999) description of better-reply security (the differences are emphasized), admissible security requires that for each non-Nash equilibrium limit admissible strategy $\sigma^{*}$ and every payoff vector limit $u^{*}$ resulting from limit admissible strategies approaching $\sigma^{*}$, some player $i$ has a limit admissible strategy yielding a payoff strictly above $u_{i}^{*}$ even if the others deviate slightly from $\sigma^{*}$ to another limit admissible strategy.

Admissible security is a relativization of better-reply security in mixed strategies and, in this way, is neither stronger nor weaker than better-reply security in mixed strategies. ${ }^{3}$ A further relativization, namely changing (b) by requiring it to hold only for non-equilibrium strategies of the game obtained by restricting players to choose limit admissible strategies, is not possible when the goal is to obtain a condition that is sufficient for the existence of limit admissible equilibria. This is shown by Example 2 which has no limit admissible equilibrium.

Note further that Example 1 satisfies (b) (vacuously) but not (a) and that Example 2 satisfies (a) but not (b). Thus, both conditions in the definition of admissible security are needed for this condition to imply the existence of limit admissible equi-

\footnotetext{
${ }^{3} \mathrm{~A}$ sufficient condition for admissible security which is stronger than better-reply security in mixed strategies is the following: If $\left(\sigma^{*}, u^{*}\right) \in \operatorname{cl}(\operatorname{graph}(u))$ and $\sigma^{*}$ is not a Nash equilibrium of $G$, then there exist $i \in N, \mu_{i} \in M_{i}^{0}$, an open neighborhood $U$ of $\sigma^{*}$ and a real number $\alpha_{i}>u_{i}^{*}$ such that $u_{i}\left(\mu_{i}, \sigma_{-i}\right) \geq \alpha_{i}$ for each $\sigma \in U$.
} 
libria. Our main result states that admissible security is indeed sufficient for the existence of limit admissible equilibria.

Theorem 1. If $G=\left(X_{i}, u_{i}\right)_{i \in N}$ is admissibly secure, then $L(G) \neq \emptyset$.

Proof. Let $G$ be admissibly secure and consider $G^{0}=\left(M_{i}^{0},\left.u_{i}\right|_{M^{0}}\right)_{i \in N}$, where $\left.u_{i}\right|_{M^{0}}$ is the restriction of $u_{i}$ to $M^{0}$ for each $i \in N$. Note that $\operatorname{cl}\left(\left\{(\sigma, u(\sigma)): \sigma \in M^{0}\right\}\right)=$ $\operatorname{cl}\left(\operatorname{graph}\left(\left.u\right|_{M^{0}}\right)\right)$ and that $U \cap M^{0}$ is open in $M^{0}$ whenever $U$ is open in $M$. Furthermore, if $\sigma^{*} \in M^{0}$ is not a Nash equilibrium of $G^{0}$, then $\sigma^{*}$ is not a Nash equilibrium of $G$. Hence, $G^{0}$ is better-reply secure. It then follows by Reny (1999, Theorem 3.1) ( $G^{0}$ is clearly a compact and own-strategy quasi-concave game) that $G^{0}$ has a Nash equilibrium $\sigma^{*} \in M^{0}$.

Suppose, in order to reach a contradiction, that $L(G)=\emptyset$. Then $\sigma^{*}$ is not a Nash equilibrium of $G$. Thus, as $\left(\sigma^{*}, u\left(\sigma^{*}\right)\right) \in \operatorname{cl}\left(\left\{(\sigma, u(\sigma)): \sigma \in M^{0}\right\}\right)$ and $G$ is admissible secure, there exists $i \in N$ and $\mu_{i} \in M_{i}^{0}$ such that $u_{i}\left(\mu_{i}, \sigma_{-i}^{*}\right)>u_{i}\left(\sigma^{*}\right)$. But this is a contradiction since $\sigma^{*}$ is a Nash equilibrium of $G^{0}$. This contradiction shows that $L(G)$ is nonempty and completes the proof.

Extensions as well as particular cases of Theorem 1 are discussed in the following remarks.

Remark 1 (Admissible correspondence security). An extension of Theorem 1 can be obtained by weakening admissible security along the lines of Reny's (2016) correspondence security. A game $G=\left(X_{i}, u_{i}\right)_{i \in N}$ is admissible correspondence secure if $C_{i} \neq \emptyset$ for each $i \in N$ and if whenever $\sigma^{*} \in M^{0}$ is not a Nash equilibrium of $G$, then there exists an open neighborhood $U$ of $\sigma^{*}$ and a closed correspondence $\psi: U \rightrightarrows M^{0}$ with nonempty and convex values such that, for each $\sigma \in U \cap M^{0}$, there is $i \in N$ for whom $u_{i}\left(\mu_{i}, \sigma_{-i}^{\prime}\right)>u_{i}(\sigma)$ for each $\sigma^{\prime} \in U \cap M^{0}$ and $\mu_{i} \in \psi_{i}\left(\sigma^{\prime}\right)$. We then have, by an argument analogous to that of Theorem 1 , that if $G=\left(X_{i}, u_{i}\right)_{i \in N}$ is admissibly correspondence secure, then $L(G) \neq \emptyset$.

Remark 2 (Admissible finite deviation property). Another extension of Theorem 1 can also be obtained by weakening admissible security along the lines of Reny's (2016) 
finite deviation property. We say that $G=\left(X_{i}, u_{i}\right)_{i \in N}$ satisfies the admissible finite deviation property if, for each $\sigma \in M^{0}$ which is not a Nash equilibrium of $G$, there exists $\mu^{1}, \ldots, \mu^{K} \in M^{0}$ and a neighborhood $U$ of $\sigma$ such that, for each $\sigma^{\prime} \in U \cap M^{0}$, there exists $i \in N$ and $k \in\{1, \ldots, K\}$ such that $u_{i}\left(\mu_{i}^{k}, \sigma_{-i}^{\prime}\right)>u_{i}\left(\sigma^{\prime}\right)$. Adapting the proof of Theorem 1 , one can show that $L(G) \neq \emptyset$ for each game with the admissible finite deviation property.

Remark 3 (Pure strategies). Our existence result extends to the case of pure strategies in games $G=\left(X_{i}, u_{i}\right)_{i \in N}$ that are own-strategy quasi-concave (i.e. $X_{i}$ is convex and $u_{i}\left(\cdot, x_{-i}\right)$ is quasi-concave for each $i \in N$ and $\left.x_{-i} \in X_{-i}\right)$ and are such that $C_{i}$ is convex for each $i \in N$. In this case, letting $C=\prod_{i \in N} C_{i}$, part (b) of the definition of admissible security is specialized to the case of pure strategies as follows: If $\left(x^{*}, u^{*}\right) \in \operatorname{cl}(\{(x, u(x)): x \in C\})$ and $x^{*}$ is not a Nash equilibrium of $G$, then there exist $i \in N, \bar{x}_{i} \in C_{i}$, an open neighborhood $U$ of $x^{*}$ and a real-number $\alpha_{i}>u_{i}^{*}$ such that $u_{i}\left(\bar{x}_{i}, x_{-i}\right) \geq \alpha_{i}$ for each $x \in U \cap C$.

The Bertrand duopoly of Example 3 illustrates. Indeed, it is a quasi-concave game in which $C_{i}$ is convex for each $i=1,2$, namely $C_{i}=[c,(1+c) / 2]$ for each $i=1,2$ (note that $(1+c) / 2$ is the monopoly quantity). Furthermore, it is admissibly secure: When $x^{*} \in C$ is not a Nash equilibrium (i.e. $x^{*} \neq(c, c)$ ) and $x_{i}^{*}=x_{j}^{*}>c$, pick player $i, \bar{x}_{i} \in\left[c, x_{j}^{*}\right)$ sufficiently close to $x_{i}^{*}$ and $U=X_{i} \times\left(\bar{x}_{i}, x_{j}^{*}\right)$; if $x_{i}^{*}>x_{j}^{*} \geq c$, pick player $j, \bar{x}_{j} \in\left(c, x_{i}^{*}\right)$ and $U=X_{j} \times\left(\bar{x}_{j}, x_{i}^{*}\right)$. Thus, in accordance with the above, the Bertrand duopoly of Example 3 has a pure-strategy limit admissible equilibrium, namely $x^{*}=(c, c)$.

In general, however, own-strategy quasi-concave games satisfying admissible security may fail to have a pure-strategy limit admissible equilibrium when $C_{i}$ fails to be convex for some $i \in N$. To illustrate, we present an example of an own-strategy quasi-concave game with continuous payoff functions that fails to have a limit admissible equilibrium. ${ }^{4}$ The game $G=\left(X_{i}, u_{i}\right)_{i \in N}$ is a variation on the matching pennies.

\footnotetext{
${ }^{4}$ Games with continuous payoff functions have pure-strategy limit admissible equilibria provided that $X_{i}$ is convex and $u_{i}\left(\cdot, x_{-i}\right)$ is concave for each $i \in N$ and $x_{-i} \in X_{-i}$; see Carbonell-Nicolau (2011a) for this and related results.
} 
Let $N=\{1,2\}$ and $X_{1}=X_{2}=[0,1]$, where $x_{i}$ is interpreted as the probability of player $i$ playing heads, $i=1,2$. Player 1 want to match his choice with that of player 2 and, thus, let

$$
u_{1}(x)=-\left|x_{1}-x_{2}\right|
$$

for each $x \in X$. As for player 2's payoff function, let, for each $x \in X$,

$$
w_{2}(x)=\left(1-2 x_{1}\right)\left(2 x_{2}-1\right)
$$

as in the standard matching pennies, and

$$
u_{2}(x)=\max \left\{w_{2}(x), 0\right\}
$$

It is easy to see that $G$ is own-strategy quasi-concave with continuous payoff functions and that it has a unique pure-strategy Nash equilibrium $x^{*}=(1 / 2,1 / 2)$. Moreover, $C_{2}=\{0,1\}$ since any $x_{2} \in[1 / 2,1)$ is weakly dominated by $x_{2}^{\prime}=1$, any $x_{2} \in(0,1 / 2)$ is weakly dominated by $x_{2}^{\prime}=0$ and neither $x_{2}=0$ nor $x_{2}=1$ are weakly dominated. Therefore, $G$ has no limit admissible equilibria in pure strategies.

Remark 4 (Sufficient conditions 1). We say that a game $G=\left(X_{i}, u_{i}\right)_{i \in N}$ is admissibly payoff secure if for each $i \in N, \varepsilon>0$ and $\sigma \in M_{i} \times M_{-i}^{0}$, there is a $\mu_{i} \in M_{i}^{0}$ and an open neighborhood $U$ of $\sigma$ such that

$$
u_{i}\left(\mu_{i}, \sigma_{-i}^{\prime}\right)>u_{i}(\sigma)-\varepsilon
$$

for each $\sigma^{\prime} \in U \cap M^{0}$. Moreover, we say that $G$ is admissibly reciprocal upper semicontinuous if for each $\left(\sigma^{*}, \alpha\right) \in \operatorname{cl}\left(\left\{(\sigma, u(\sigma)): \sigma \in M^{0}\right\}\right) \backslash\left\{(\sigma, u(\sigma)): \sigma \in M^{0}\right\}$, there exists $i \in N$ and $\mu_{i} \in M_{i}$ such that $u_{i}\left(\mu_{i}, \sigma_{-i}^{*}\right)>\alpha_{i}$. Analogously to Bagh and Jofre (2006, Proposition 1), we obtain that if $G$ is admissibly payoff secure and admissibly reciprocal upper semi-continuous, then $G$ is admissibly secure.

Remark 5 (Continuous games). Games with continuous payoff functions, considered in Simon and Stinchcombe (1995), are easily seen to be admissible secure. Indeed, admissible reciprocal upper semi-continuity is trivially satisfied as $\operatorname{cl}(\{(\sigma, u(\sigma)): \sigma \in$ $\left.\left.M^{0}\right\}\right) \backslash\left\{(\sigma, u(\sigma)): \sigma \in M^{0}\right\}$ is empty. Furthermore, for admissible payoff security, 
it suffices to show that, against any $\sigma \in M$, each player $i$ has a best-reply in $M_{i}^{0}$. To see that this property holds, let $i \in N$ and $\sigma \in M$ be given. Letting $j \neq i$ and $\left\{x_{k, j}\right\}_{k=1}^{\infty}$ be dense in $X_{j}$, define $\bar{\sigma}_{j}=\sum_{k=1}^{\infty} \frac{1}{2^{k}} \delta_{x_{k, j}} \in M_{j}$ and $\sigma_{k, j}=\frac{1}{k} \bar{\sigma}_{j}+$ $\left(1-\frac{1}{k}\right) \sigma_{j}$ for each $k \in \mathbb{N}$. Fix $k \in \mathbb{N}$ and, using the continuity of $u_{i}$, let $x_{k, i} \in X_{i}$ be such that $u_{i}\left(x_{k, i}, \sigma_{k,-i}\right)=\max _{\sigma_{i} \in M_{i}} u_{i}\left(\sigma_{i}, \sigma_{k,-i}\right)$. If $x_{k, i} \notin C_{i}$, then letting $\mu_{i} \in$ $M_{i}$ weakly dominate $x_{k, i}$, we have that $u_{i}\left(x_{k, i}, \sigma_{k,-i}\right)<u_{i}\left(\mu_{i}, \sigma_{k,-i}\right)$ as $\tau_{\sigma_{k,-i}}$ gives strictly positive probability to each open subset of $X_{-i}$; but this is a contradiction to $u_{i}\left(x_{k, i}, \sigma_{k,-i}\right)=\max _{\sigma_{i} \in M_{i}} u_{i}\left(\sigma_{i}, \sigma_{k,-i}\right)$. Thus, $x_{k, i} \in C_{i}$ for each $k \in \mathbb{N}$. Since $C_{i}$ is compact, we may assume that $\left\{x_{k, i}\right\}_{k=1}^{\infty}$ converges and let $x_{i}=\lim _{k} x_{k, i}$. Then $x_{i} \in C_{i}$ and the continuity of $u_{i}$, together with $\sigma_{k,-i} \rightarrow \sigma_{-i}$, implies that $u_{i}\left(x_{i}, \sigma_{-i}\right)=$ $\max _{\sigma_{i} \in M_{i}} u_{i}\left(\sigma_{i}, \sigma_{-i}\right)$.

Remark 6 (A related approach). One way of obtaining continuous games is to endow players' strategy spaces with Fudenberg and Levine's (1986) "most utility difference it can make to anyone" pseudo-metric, defined by

$$
p\left(x_{i}, x_{i}^{\prime}\right)=\max _{j \in N} \sup _{x_{-i} \in X_{-i}}\left|u_{j}\left(x_{i}, x_{-i}\right)-u_{j}\left(x_{i}^{\prime}, x_{-i}\right)\right|
$$

for each $x_{i}, x_{i}^{\prime} \in X_{i}$ in a game $G=\left(X_{i}, u_{i}\right)_{i \in N}$. In general, however, players' strategy spaces may fail to be compact. ${ }^{5}$ This difficulty can sometimes be overcome in a way that allows for existence of Nash equilibrium by compactification as shown by Harris, Stinchcombe, and Zame (2005).

We leave as open the question of under what conditions can this approach yield an existence results for limit admissible equilibria. Here we just remark that there are games to which our approach can be applied but not that of Harris, Stinchcombe, and Zame (2005). Such an example is provided by their Example 2: Let $G=\left(X_{i}, u_{i}\right)_{i \in N}$ be a 2-player game on the unit square (i.e. $N=\{1,2\}$ and $X_{1}=X_{2}=[0,1]$ ) with the following payoff functions: For each $x \in X$ and $i \in N$,

$$
u_{i}(x)= \begin{cases}x_{1} & \text { if } x_{1}=x_{2} \\ 0 & \text { otherwise }\end{cases}
$$

\footnotetext{
${ }^{5}$ These observations provide an alternative view on Examples 1 and 2.
} 
for each $x \in X$. In this game, $C_{i}=X_{i}$ for each $i \in N$, hence the set of limit admissible equilibria coincides with the set of Nash equilibria. As Harris, Stinchcombe, and Zame (2005) show, this game is not nearly compact and continuous, hence their results do not apply. In contrast, the condition in Remark 1 holds in pure strategies (because $C_{i}=X_{i}$ for each $i \in N$, this is just Reny's (2016) correspondence security) and, as discussed in Remark 3 hence, $G$ has a (limit admissible) equilibrium in pure strategies.

Remark 7 (Related results). The existence of a limit admissible equilibrium can be obtained from some results in Carbonell-Nicolau (2011b), namely from Theorem 4 and also by combining Theorem 7 and Remark 6 in that paper. Under the assumptions of Theorem 7 in Carbonell-Nicolau (2011b), both admissible payoff security and admissible reciprocal upper semicontinuity hold, hence, the existence of a limit admissible equilibrium follows by Remark 4 and Theorem 1 above (this argument makes Remark 6 in Carbonell-Nicolau (2011b) redundant). Under the assumptions of Theorem 4 in Carbonell-Nicolau (2011b), better-reply security in mixed strategies holds and so does admissible reciprocal upper semicontinuity. However, it is unclear whether or not admissible payoff security holds. This is the case even under the assumptions of Carbonell-Nicolau's (2011b) Theorem 4 which imply payoff security, i.e., it is the case that for each $i \in N, \varepsilon>0$ and $\sigma \in M$, there is $\mu_{i} \in M_{i}$ and an open neighborhood $U$ of $\sigma$ such that $u_{i}\left(\mu_{i}, \sigma_{-i}^{\prime}\right)>u_{i}(\sigma)-\varepsilon$ for each $\sigma^{\prime} \in U$; the difficulty is to conclude that $\mu_{i}$ can be taken to be an element of $M_{i}^{0}$ as required by admissible payoff security. On the other end, our Theorem 1 does not require any of the conditions imposed in Carbonell-Nicolau's (2011b) results.

Remark 8 (Sufficient conditions 2). Carbonell-Nicolau's (2011b) Theorem 4 described in the previous remark can be seen as providing conditions that, when added to better-reply security in mixed strategies, imply the existence of Nash equilibria with additional structure. Here we ask: What additional conditions need to be added to better-reply security in mixed strategies for a game to have a limit admissible equilibrium? One such condition is: For each $i \in N$ and $\sigma_{i} \in M_{i} \backslash M_{i}^{0}$, there exists $\sigma_{i}^{0} \in M_{i}^{0}$ such that $u_{i}\left(\sigma_{i}^{0}, x_{-i}\right) \geq u_{i}\left(\sigma_{i}, x_{-i}\right)$ for each $x_{-i} \in X_{-i}$; this is a weakening of 
the requirement that each strategy outside $M_{i}^{0}$ be weakly dominated by a strategy in $M_{i}^{0}$. It is easy to see that any game satisfying this condition and better-reply security in mixed strategies is admissibly secure.

Remark 9 (Bertrand duopoly). The conditions in the previous remark apply to the Bertrand duopoly of Example 3. It is well known that better-reply security in mixed strategies holds for this example. Moreover, for each $i \in N$ and $\sigma_{i} \in M_{i} \backslash M_{i}^{0}$, there exists $\sigma_{i}^{0} \in M_{i}^{0}$ such that $u_{i}\left(\sigma_{i}^{0}, x_{-i}\right) \geq u_{i}\left(\sigma_{i}, x_{-i}\right)$ for each $x_{-i} \in X_{-i}$. To see this, recall that $C_{i}=[c,(1+c) / 2]$, define $f: X_{i} \rightarrow C_{i}$ by setting, for each $x_{i} \in X_{i}, f\left(x_{i}\right)=c$ when $x_{i}<c, f\left(x_{i}\right)=(1+c) / 2$ when $x_{i}>(1+c) / 2$ and $f\left(x_{i}\right)=x_{i}$ otherwise and set $\sigma_{i}^{0}=\sigma_{i} \circ f^{-1} \in M_{i}^{0}$. For each $x_{-i} \in X_{-i}$, we have that $u_{i}\left(x_{i}, x_{-i}\right) \leq 0=u_{i}\left(c, x_{-i}\right)$ for each $x_{i}<c$ and $u_{i}\left(x_{i}, x_{-i}\right) \leq u_{i}\left((1+c) / 2, x_{-i}\right)$ for each $x_{i}>(1+c) / 2$. Hence, $u_{i}\left(\sigma_{i}^{0}, x_{-i}\right)-u_{i}\left(\sigma_{i}, x_{-i}\right)=\int_{[0, c)}\left(u_{i}\left(c, x_{-i}\right)-u_{i}\left(x_{i}, x_{-i}\right)\right) \mathrm{d} \sigma_{i}\left(x_{i}\right)+\int_{((1+c) / 2,1]}\left(u_{i}((1+\right.$ c) $\left.\left./ 2, x_{-i}\right)-u_{i}\left(x_{i}, x_{-i}\right)\right) \mathrm{d} \sigma_{i}\left(x_{i}\right) \geq 0$. Thus, Theorem 1 applies.

Remark 10 (An extension). In Theorem 1, $C_{i}$ is the closure of the set of undominated strategies for each $i \in N$. More generally, the conclusion of Theorem 1 holds for any nonempty, closed subset of $X_{i}$ as follows. Let $G=\left(X_{i}, u_{i}\right)_{i \in N}$ be a game and, for each $i \in N, \tilde{C}_{i}$ be a nonempty, closed subset of $X_{i}$. Define $\tilde{M}_{i}=\left\{\sigma_{i} \in M_{i}: \sigma_{i}\left(\tilde{C}_{i}\right)=1\right\}$

for each $i \in N$ and $\tilde{M}=\prod_{i \in N} \tilde{M}_{i}$. Then $G$ has a Nash equilibrium in $\tilde{M}$ provided that the following condition analogous to admissible security holds: If $\left(\sigma^{*}, u^{*}\right) \in$ $\operatorname{cl}(\{(\sigma, u(\sigma)): \sigma \in \tilde{M}\})$ and $\sigma^{*}$ is not a Nash equilibrium of $G$, then there exist $i \in N, \mu_{i} \in \tilde{M}_{i}$, an open neighborhood $U$ of $\sigma^{*}$ and a real number $\alpha_{i}>u_{i}^{*}$ such that $u_{i}\left(\mu_{i}, \sigma_{-i}\right) \geq \alpha_{i}$ for each $\sigma \in U \cap \tilde{M}$.

\section{An applications: Bertrand-Edgeworth duopoly with strictly convex costs}

We consider a Bertrand-Edgeworth duopoly with strictly convex costs as an economic application of our main result, Theorem 1. We depart from the standard formalization of Bertrand competition considered in Andersson, Argenton, and Weibull (2014) and 
Bich (2016) because the assumption that the firm posting the lowest price serves the entire demand leads to difficulties in the presence of strictly convex costs. This is so because firms may prefer to tie to reduce the quantity produced. For this reason, it is more appropriate to allow firms to choose the quantity they want to supply. We allow for this by allowing each firm to choose a price and the maximum production level it is willing to produce, i.e. an endogenous capacity. Our formalization is thus analogous to that of Dasgupta and Maskin (1986, Section 2.2) where each firm has a capacity but which is exogenously given. Our formalization is closer to that of Maskin (1986) where firms choose both prices and quantities, and firms produce to order, i.e., produce only after the entire price profile has been observed.

As in Dasgupta and Maskin (1986), there is a market for a single commodity with a continuum of consumers represented by the unit interval $[0,1]$. Consumers are identical, and the representative consumer's demand for the commodity is a function $d: \mathbb{R}_{+} \rightarrow \mathbb{R}_{+}$such that there exists $\bar{p}>0$ satisfying $d(p)>0, d^{\prime}(p)<0$ and $d^{\prime \prime}(p) \leq 0$ for all $p \leq \bar{p}, d(p)=0$ for all $p \geq \bar{p}$ and $d$ is $C^{2}$ in $[0, \bar{p}]$. There is a $C^{2}$ cost function $c: \mathbb{R}_{+} \rightarrow \mathbb{R}_{+}$satisfying $c(0)=0, \bar{p}>c^{\prime}(0)$ and, for each $q>0, c^{\prime}(q)>0$ and $c^{\prime \prime}(q)>0$.

There are two firms. Each firm $i=1,2$ chooses a price $p_{i}$ and a capacity $s_{i}$, the latter being the maximum amount the firm is willing to produce. Let $P=[0, \bar{p}]$ and $S=[0, d(0)]$.

If one firm offers a price $p$ lower than the price $p^{\prime}$ offered by the other firm, then it serves the entire market up to its capacity $s$. A fraction $\frac{(d(p)-s)^{+}}{d(p)}=\frac{\max \{d(p)-s, 0\}}{d(p)}$ of consumers is not served and each of these consumers demands $d\left(p^{\prime}\right)$ from the firm offering the highest price. When both firms set the same price, then the demand at the common price is split by each firm up to its capacity.

In the latter case, several allocations are possible and we will focus on allocations that maximize the total profits in the market. Formally, the quantity produced by firms is described by the correspondence $\Phi:(P \times S)^{2} \rightrightarrows \mathbb{R}_{+}^{2}$ defined by setting, for 
each $x \in(P \times S)^{2}$,

$$
\Phi(x)= \begin{cases}\left(\min \left\{d\left(p_{1}\right), s_{1}\right\}, \min \left\{\frac{\left(d\left(p_{1}\right)-s_{1}\right)^{+}}{d\left(p_{1}\right)} d\left(p_{2}\right), s_{2}\right\}\right) & \text { if } p_{1}<p_{2}, \\ \left\{\phi \in \mathbb{R}_{+}^{2}: \phi_{1}+\phi_{2} \leq d\left(p_{1}\right), \phi_{i} \leq s_{i}\right. \text { and } & \\ \left.\left[d\left(p_{1}\right)-\phi_{1}-\phi_{2}\right]\left[s_{i}-\phi_{i}\right]=0 \text { for each } i=1,2\right\} & \text { if } p_{1}=p_{2}, \\ \left(\min \left\{\frac{\left(d\left(p_{2}\right)-s_{2}\right)^{+}}{d\left(p_{2}\right)} d\left(p_{1}\right), s_{1}\right\}, \min \left\{d\left(p_{2}\right), s_{2}\right\}\right) & \text { if } p_{1}>p_{2} .\end{cases}
$$

When prices are different, these quantities are the same as in both Dasgupta and Maskin (1986) and Maskin (1986) (with the proportional rationing rule in the latter). However, we rule out the possibility that a firm produces less than its capacity when there is unfulfilled demand (i.e. $\phi_{1}+\phi_{2}<d\left(p_{1}\right)$ and $\phi_{i}<s_{i}$ for some $i$ is not possible); in contrast this is allowed in both Dasgupta and Maskin (1986) and Maskin (1986).

We then let $\phi:(P \times S)^{2} \rightrightarrows \mathbb{R}_{+}^{2}$ be measurable and such that, for each $x \in(P \times S)^{2}$, $\phi(x)$ solves

$$
\max _{\phi \in \Phi(x)} \sum_{i=1}^{2}\left(p_{i} \phi_{i}-c\left(\phi_{i}\right)\right) .
$$

Players' payoffs are then

$$
u_{i}(x)=p_{i} \phi_{i}(x)-c\left(\phi_{i}(x)\right)
$$

for each $i \in\{1,2\}$ and $x \in(P \times S)^{2}$. Our results, however, extend to a broad class of Bertrand-Edgeworth games obtained by changing the function $\phi$; see Remark 11 below.

The function $\phi$ and, therefore, players' payoff functions can be explicitly computed.

Lemma 1. For each $x \in(P \times S)^{2}$ with $p_{1}=p_{2}$,

$$
\phi(x)= \begin{cases}\left(s_{1}, s_{2}\right) & \text { if } s_{1}+s_{2}<d\left(p_{1}\right), \\ \left(s_{1}, d\left(p_{1}\right)-s_{1}\right) & \text { if } 2 \min \left\{s_{1}, s_{2}\right\}<d\left(p_{1}\right) \leq s_{1}+s_{2} \text { and } s_{1}<s_{2}, \\ \left(d\left(p_{1}\right)-s_{2}, s_{2}\right) & \text { if } 2 \min \left\{s_{1}, s_{2}\right\}<d\left(p_{1}\right) \leq s_{1}+s_{2} \text { and } s_{1}>s_{2}, \\ \left(d\left(p_{1}\right) / 2, d\left(p_{1}\right) / 2\right) & \text { if } d\left(p_{1}\right) \leq 2 \min \left\{s_{1}, s_{2}\right\} .\end{cases}
$$

Proof. Let $x \in(P \times S)^{2}$ be such that $p_{1}=p_{2}$ and let $p$ denote this common value. The definition of $\Phi(x)$ implies that $\phi(x)=\left(s_{1}, s_{2}\right)$ if $s_{1}+s_{2}<d(p)$. Moreover, the 
definition of $\Phi(x)$ also implies that $\phi_{1}+\phi_{2}=d(p)$ when $s_{1}+s_{2} \geq d(p)$. Hence, in this case, $\phi(x)$ is the solution to

$$
\begin{aligned}
& \max _{\phi_{1}, \phi_{2}} p\left(\phi_{1}+\phi_{2}\right)-c\left(\phi_{1}\right)-c\left(\phi_{2}\right) \\
& \text { subject to } \phi_{1}+\phi_{2}=d(p), 0 \leq \phi_{1} \leq s_{1}, 0 \leq \phi_{2} \leq s_{2} .
\end{aligned}
$$

This problem can be rewritten as

$$
\begin{aligned}
& \max _{\phi_{1}} p d(p)-c\left(\phi_{1}\right)-c\left(d(p)-\phi_{1}\right) \\
& \text { subject to } \max \left\{0, d(p)-s_{2}\right\} \leq \phi_{1} \leq \min \left\{s_{1}, d(p)\right\} .
\end{aligned}
$$

The unconstrained solution is $\phi_{1}$ such that $c^{\prime}\left(\phi_{1}\right)=c^{\prime}\left(d(p)-\phi_{1}\right)$, i.e. $\phi_{1}=d(p) / 2$. Thus, $\phi(x)=(d(p) / 2, d(p) / 2)$ when $\max \left\{0, d(p)-s_{2}\right\} \leq d(p) / 2 \leq \min \left\{s_{1}, d(p)\right\}$, i.e. when $d(p) \leq 2 \min \left\{s_{1}, s_{2}\right\}$.

Finally, consider the case $2 \min \left\{s_{1}, s_{2}\right\}<d(p) \leq s_{1}+s_{2}$. Clearly, $s_{1} \neq s_{2}$; for concreteness, assume that $s_{1}<s_{2}$. Then $\min \left\{s_{1}, d(p)\right\}=s_{1}$. If $d(p)=s_{1}+s_{2}$ or $s_{1}=0$, then $\max \left\{0, d(p)-s_{2}\right\}=s_{1}$ and, hence, $\phi(x)=\left(s_{1}, d(p)-s_{1}\right)$. Finally, if $d(p)<s_{1}+s_{2}$ and $s_{1}>0$, there is $\max \left\{0, d(p)-s_{2}\right\}<\phi_{1}<s_{1}$ and, at any such $\phi_{1}$, the derivative of the objective function is $-c^{\prime}\left(\phi_{1}\right)+c^{\prime}\left(d(p)-\phi_{1}\right)>0$ since $\phi_{1}<s_{1}<d(p) / 2$. This shows that $\phi(x)=\left(s_{1}, d(p)-s_{1}\right)$.

While in the above we allowed firms to choose any capacity in $S$, some choices are easily seen to be redundant. Indeed, note that for each $i=1,2, x=\left(p_{i}, s_{i}, p_{j}, s_{j}\right)$ and $\phi \in \Phi(x)$, we have that $\phi_{i} \leq d\left(p_{i}\right)$. Furthermore, if $s_{i}>d\left(p_{i}\right)$ and $x^{\prime}=$ $\left(p_{i}, d\left(p_{i}\right), p_{j}, s_{j}\right)$, then $\phi_{i}(x)=\phi_{i}\left(x^{\prime}\right)$ (this can easily be seen using the definition of $\Phi$ and Lemma 1). Hence, we may assume that $s_{i} \leq d\left(p_{i}\right)$ for each $i=1,2$ and $p_{i} \in P$. In particular, $s_{i}=0$ whenever $p_{i}=\bar{p}$. We then let $X_{i}=\left\{\left(p_{i}, s_{i}\right) \in P \times S: s_{i} \in\left[0, d\left(p_{i}\right)\right]\right\}$ for each $i \in N$.

Let $G_{B}=\left(X_{1}, X_{2}, u_{1}, u_{2}\right)$ be the game just defined. The following is our main result of this section.

Theorem 2. The game $G_{B}$ is admissibly secure and, thus, $L\left(G_{B}\right) \neq \emptyset$. 
Proceeding to the proof of Theorem 2, we start by characterizing the set $C_{i}$ for each $i \in\{1,2\}$. Define, for each $p \in P, \phi^{*}(p)$ to be the unique solution to

$$
\max _{0 \leq \phi \leq d(p)}(p \phi-c(\phi))
$$

Note that the uniqueness of the solution follows because $c$ is strictly convex. Define $m: P \rightarrow \mathbb{R}_{+}$by setting

$$
m(p)=p \phi^{*}(p)-c\left(\phi^{*}(p)\right)
$$

for each $p \in P$. It follows by the maximum theorem (e.g. Aliprantis and Border (2006, Theorem 17.31, p. 570)) that $m$ and $\phi^{*}$ are continuous. Further properties of $m$ and $\phi^{*}$ are described in the following lemma.

Lemma 2. The following holds:

1. There exists a unique $\hat{p} \in P$ such that $c^{\prime}(d(\hat{p}))=\hat{p}$. Moreover, $c^{\prime}(0)<\hat{p}<\bar{p}$ and $p-c^{\prime}(d(p))>0$ for each $p>\hat{p}$.

2. For each $p \in P$,

$$
\phi^{*}(p)= \begin{cases}0 & \text { if } p \leq c^{\prime}(0) \\ \left(c^{\prime}\right)^{-1}(p) & \text { if } c^{\prime}(0)<p<\hat{p} \\ d(p) & \text { if } p \geq \hat{p} .\end{cases}
$$

3. The function $m$ has a unique maximizer $p^{*} \in(\hat{p}, \bar{p})$. Furthermore, $m$ is strictly increasing in $\left[c^{\prime}(0), p^{*}\right]$ and strictly decreasing in $\left[p^{*}, \bar{p}\right]$.

For example, if $c(q)=q^{2}$ for each $q \in \mathbb{R}_{+}$and $d(p)=1-p$ for each $p \in P$, then $\hat{p}=2 / 3$,

$$
\begin{gathered}
\phi^{*}(p)= \begin{cases}p / 2 & \text { if } p<2 / 3, \\
1-p & \text { if } p \geq 2 / 3,\end{cases} \\
m(p)= \begin{cases}p^{2} / 4 & \text { if } p<2 / 3, \\
3 p-1-2 p^{2} & \text { if } p \geq 2 / 3,\end{cases}
\end{gathered}
$$

and $p^{*}=3 / 4$. 
Proof of Lemma 2. We start with the proof of part 1. Define, for each $p \in P, f(p)=$ $p-c^{\prime}(d(p))$. We have that $f^{\prime}(p)=1-c^{\prime \prime}(d(p)) d^{\prime}(p) \geq 1$ for each $p \in P$; hence $f$ is strictly increasing. Moreover, $f$ is continuous, $f(0)=-c^{\prime}(d(0))<0$ and $f(\bar{p})=$ $\bar{p}-c^{\prime}(0)>0$. Thus, there exists $\hat{p} \in(0, \bar{p})$ such that $f(\hat{p})=0$. Since $f$ is strictly increasing, $\hat{p}$ is unique and $p-c^{\prime}(d(p))>0$ for each $p>\hat{p}$. Finally, $\hat{p}<\bar{p}$ implies that $d(\hat{p})>0$ and, therefore, $\hat{p}=c^{\prime}(d(\hat{p}))>c^{\prime}(0)$.

We next turn to the proof of part 2 of the lemma. Let, for each $p \in P$ and $\phi \in \mathbb{R}_{+}$, $f(\phi ; p)=p \phi-c(\phi)$. If $p \leq c^{\prime}(0)$, then $f^{\prime}(\phi ; p)=p-c^{\prime}(\phi)<p-c^{\prime}(0) \leq 0$ for each $\phi>0$; hence, $\phi^{*}(p)=0$.

If $p \geq \hat{p}$, then $f^{\prime}(\phi ; p)=p-c^{\prime}(\phi)>p-c^{\prime}(d(p)) \geq 0$ for each $\phi<d(p)$, where the last inequality follows by part 1 ; hence, $\phi^{*}(p)=d(p)$.

Finally, when $p \in\left(c^{\prime}(0), \hat{p}\right)$, the first order condition gives $p=c^{\prime}\left(\phi^{*}(p)\right)$.

We now establish part 3 of the lemma. Note first that $m(p)=0$ for each $p \leq c^{\prime}(0)$ by part 2 . Next, note that $m$ is strictly increasing in $\left[c^{\prime}(0), \hat{p}\right]$. Indeed, for each $p \in\left(c^{\prime}(0), \hat{p}\right), m$ is differentiable at $p$ and, using part 2 ,

$$
m^{\prime}(p)=\phi^{*}(p)+\frac{p-c^{\prime}\left(\phi^{*}(p)\right)}{c^{\prime \prime}\left(\phi^{*}(p)\right)}=\phi^{*}(p)>0 .
$$

For each $p \in(\hat{p}, \bar{p}), \phi^{*}(p)=d(p)$ by part 2 . Hence,

$$
\begin{aligned}
& m^{\prime}(p)=d(p)+\left[p-c^{\prime}(d(p))\right] d^{\prime}(p), \text { and } \\
& m^{\prime \prime}(p)=d^{\prime}(p)\left[2-c^{\prime \prime}(d(p)) d^{\prime}(p)\right]+d^{\prime \prime}(p)\left[p-c^{\prime}(d(p))\right] .
\end{aligned}
$$

Hence, using part 1, it follows that $\lim _{p \rightarrow \hat{p}} m^{\prime}(p)=d(\hat{p})+\left[\hat{p}-c^{\prime}(d(\hat{p}))\right] d^{\prime}(\hat{p})=d(\hat{p})>0$, $\lim _{p \rightarrow \bar{p}} m^{\prime}(p)=\left[\bar{p}-c^{\prime}(d(\bar{p}))\right] d^{\prime}(\bar{p})<0$ and $m^{\prime \prime}(p)<0$ for each $p \in(\hat{p}, \bar{p})$. Thus, there exists a unique $p^{*} \in(\hat{p}, \bar{p})$ such that $m^{\prime}\left(p^{*}\right)=0, m^{\prime}(p)>0$ for each $p \in\left(\hat{p}, p^{*}\right)$ and $m^{\prime}(p)<0$ for each $p \in\left(p^{*}, \bar{p}\right)$. The conclusion follows.

We use the above lemma to characterize the interior of the set of weakly dominated strategies.

Lemma 3. For each $i=1,2$,

$$
C_{i}=\left\{\left(p_{i}, s_{i}\right) \in X_{i}: p_{i} \in\left[c^{\prime}(0), p^{*}\right], s_{i}=\phi^{*}\left(p_{i}\right)\right\} .
$$


Lemma 3 is obtained by combining Lemmas $4-8$ below.

Lemma 4. If $i \in\{1,2\}, x=\left(p_{1}, s_{1}, p_{2}, s_{2}\right) \in(P \times S)^{2}$ and $x^{\prime}=\left(p_{1}^{\prime}, s_{1}^{\prime}, p_{2}^{\prime}, s_{2}^{\prime}\right) \in$ $(P \times S)^{2}$ are such that $p_{1}^{\prime}=p_{1}, p_{2}^{\prime}=p_{2}, s_{j}^{\prime}=s_{j}$ and $s_{i}^{\prime}>s_{i}$, then either $\phi_{i}(x)=\phi_{i}\left(x^{\prime}\right)$ or $\phi_{i}(x)=s_{i}<\phi_{i}\left(x^{\prime}\right)$.

Proof. We have that:

$$
\begin{aligned}
& \phi_{i}(x)=\min \left\{d\left(p_{i}\right), s_{i}\right\} \leq \min \left\{d\left(p_{i}\right), s_{i}^{\prime}\right\}=\phi_{i}\left(x^{\prime}\right) \text { if } p_{i}<p_{j}, \\
& \phi_{i}(x)=\min \left\{\frac{\left(d\left(p_{j}\right)-s_{j}\right)^{+}}{d\left(p_{j}\right)} d\left(p_{i}\right), s_{i}\right\} \leq \min \left\{\frac{\left(d\left(p_{j}\right)-s_{j}\right)^{+}}{d\left(p_{j}\right)} d\left(p_{i}\right), s_{i}^{\prime}\right\}=\phi_{i}\left(x^{\prime}\right) \text { if } p_{i}>p_{j}, \\
& \phi_{i}(x)=s_{i}<s_{i}^{\prime}=\phi_{i}\left(x^{\prime}\right) \text { if } p_{i}=p_{j} \text { and } d\left(p_{1}\right) \geq s_{i}^{\prime}+s_{j} .
\end{aligned}
$$

In fact, in the above three cases, we have that $\phi_{i}(x)=s_{i}$ whenever $\phi_{i}(x)<\phi_{i}\left(x^{\prime}\right)$.

We next consider the two remaining cases. If $p_{i}=p_{j}$ and $s_{1}+s_{2} \leq d\left(p_{1}\right)<s_{i}^{\prime}+s_{j}$, then $\phi_{i}(x)=s_{i}$ and $\phi_{i}\left(x^{\prime}\right)=d\left(p_{1}\right)-\phi_{j}\left(x^{\prime}\right) \geq d\left(p_{1}\right)-s_{j} \geq s_{i}=\phi_{i}(x)$.

Suppose next that $d\left(p_{1}\right)<s_{1}+s_{2}$. If $d\left(p_{1}\right) \leq 2 \min \left\{s_{1}, s_{2}\right\}$, then $\phi_{i}(x)=\phi_{i}\left(x^{\prime}\right)=$ $d\left(p_{1}\right) / 2$. If $2 \min \left\{s_{1}, s_{2}\right\}<d\left(p_{1}\right) \leq 2 \min \left\{s_{i}^{\prime}, s_{j}\right\}$, it cannot be that $s_{j}=\min \left\{s_{1}, s_{2}\right\}$. Hence, $s_{i}<s_{j}$ and, thus, $s_{i}<d\left(p_{1}\right) / 2$. As $\phi_{i}(x)=s_{i}$ and $\phi_{i}\left(x^{\prime}\right)=d\left(p_{1}\right) / 2$, the conclusion of the lemma follows. Finally, if $2 \min \left\{s_{i}^{\prime}, s_{j}\right\}<d\left(p_{1}\right)$, then $s_{1} \neq s_{2}$, $s_{i}^{\prime} \neq s_{j}$ and it cannot be that $s_{i}<s_{j}<s_{i}^{\prime}$ (otherwise, $s_{j}=\min \left\{s_{i}^{\prime}, s_{j}\right\}<d\left(p_{1}\right) / 2$ and $s_{1}+s_{2}<2 s_{j}<d\left(p_{1}\right)$ contradicting $\left.d\left(p_{1}\right)<s_{1}+s_{2}\right)$. But then $\phi_{i}(x)=\phi_{i}\left(x^{\prime}\right)$. This completes the proof of the lemma.

We note that the set $C_{i}$ is independent of the sharing rule provided that the conclusion of the previous lemma holds, i.e. from this point onwards we shall only use Lemma 4 and not the explicit characterization of $\phi$ given in Lemma 1 above.

The next result shows that a capacity choice different from $\phi^{*}\left(p_{i}\right)$ when choosing a price level $p_{i}$ is weakly dominated for $i$.

Lemma 5. For each $i \in\{1,2\}$ and $\left(p_{i}, s_{i}\right) \in X_{i}$, if $s_{i} \neq \phi^{*}\left(p_{i}\right)$, then $\left(p_{i}, s_{i}\right)$ is weakly dominated for $i$ by $\left(p_{i}, \phi^{*}\left(p_{i}\right)\right)$.

Proof. Let $i \in\{1,2\},\left(p_{i}, s_{i}\right) \in X_{i}$ and $s_{i} \neq \phi^{*}\left(p_{i}\right)$. Let $j \neq i,\left(p_{j}, s_{j}\right) \in X_{j}$, $x=\left(p_{i}, \phi^{*}\left(p_{i}\right), p_{j}, s_{j}\right)$ and $x^{\prime}=\left(p_{i}, s_{i}, p_{j}, s_{j}\right)$. We clearly have $u_{i}(x)=u_{i}\left(x^{\prime}\right)$ if $\phi_{i}(x)=$ 
$\phi_{i}\left(x^{\prime}\right)$. Assuming $\phi_{i}(x) \neq \phi_{i}\left(x^{\prime}\right)$, Lemma 4 implies that $\phi_{i}(x)=\phi^{*}\left(p_{i}\right)<\phi_{i}\left(x^{\prime}\right)$ if $s_{i}>\phi^{*}\left(p_{i}\right)$ and hence $u_{i}(x)>u_{i}\left(x^{\prime}\right)$ by the definition of $\phi^{*}\left(p_{i}\right)$; furthermore, in the case $s_{i}<\phi^{*}\left(p_{i}\right)$, we have that $\phi_{i}(x)=s_{i}<\phi_{i}\left(x^{\prime}\right) \leq \phi^{*}\left(p_{i}\right)$ and thus $u_{i}(x)>u_{i}\left(x^{\prime}\right)$ by the definition of $\phi^{*}\left(p_{i}\right)$ and the strict concavity of $\phi \mapsto p_{i} \phi-c(\phi)$.

Recall that $s_{i} \leq d\left(p_{i}\right)$ and $d(\bar{p})=0$; thus, $s_{i} \neq \phi^{*}\left(p_{i}\right)$ implies that $p_{i}<\bar{p}$. Letting $p_{j}=\bar{p}$, it then follows that $p_{i}<p_{j}$ and, hence, $\phi_{i}(x)=\min \left\{d\left(p_{i}\right), \phi^{*}\left(p_{i}\right)\right\}=\phi^{*}\left(p_{i}\right)$ and $\phi_{i}\left(x^{\prime}\right)=\min \left\{d\left(p_{i}\right), s_{i}\right\}=s_{i}$ (recall also that $\left.\phi^{*}\left(p_{i}\right) \leq d\left(p_{i}\right)\right)$. Thus, $u_{i}(x)>u_{i}\left(x^{\prime}\right)$ by the definition of $\phi^{*}\left(p_{i}\right)$. This completes the proof.

It follows from Lemma 5 that $C_{i} \subseteq\left\{\left(p, \phi^{*}(p)\right): p \in P\right\}$ (recall that $\phi^{*}$ is continuous). The following lemma shows that $C_{i} \subseteq\left\{\left(p_{i}, s_{i}\right) \in X_{i}: p_{i} \geq c^{\prime}(0)\right\}$.

Lemma 6. For each $i \in\{1,2\}$, if $p_{i}<c^{\prime}(0)$, then $\left(p_{i}, \phi^{*}\left(p_{i}\right)\right)$ is weakly dominated for $i$ by $\left(\hat{p}, \phi^{*}(\hat{p})\right)$.

Proof. Let $i=1$ without loss of generality and $p_{1}<c^{\prime}(0)$. Since $\phi^{*}\left(p_{1}\right)=0$, it follows that $u_{1}\left(p_{1}, \phi^{*}\left(p_{1}\right), p_{2}, s_{2}\right)=0$ for each $\left(p_{2}, s_{2}\right) \in X_{2}$. We have that $u_{1}\left(\hat{p}, \phi^{*}(\hat{p}), p_{2}, s_{2}\right) \geq$ 0 for each $\left(p_{2}, s_{2}\right) \in X_{2}$ and, when $p_{2}>\hat{p}, u_{1}\left(\hat{p}, \phi^{*}(\hat{p}), p_{2}, s_{2}\right)=m(\hat{p})>0$.

The following lemma restrict $C_{i}$ further by showing that $C_{i} \subseteq\left\{\left(p_{i}, s_{i}\right) \in X_{i}: p_{i} \leq\right.$ $\left.p^{*}\right\}$.

Lemma 7. For each $i \in\{1,2\}$, if $p_{i}>p^{*}$, then $\left(p_{i}, \phi^{*}\left(p_{i}\right)\right)$ is weakly dominated for $i$ by $\left(p^{*}, \phi^{*}\left(p^{*}\right)\right)$.

Proof. Let $i=1$ without loss of generality and $p_{1}>p^{*}$. Lemma 2.2 implies that $\phi^{*}\left(p^{*}\right)=d\left(p^{*}\right)$ and $\phi^{*}\left(p_{1}\right)=d\left(p_{1}\right)$. Fix $\left(p_{2}, s_{2}\right) \in X_{2}$ and let $x^{*}=\left(p^{*}, d\left(p^{*}\right), p_{2}, s_{2}\right)$, $x^{\prime}=\left(p_{1}, d\left(p_{1}\right), p_{2}, s_{2}\right)$ and $\gamma=u_{1}\left(x^{*}\right)-u_{1}\left(x^{\prime}\right)$.

We consider three cases. The first case is when $p_{2}<p^{*}$. In this case, we have that $\phi_{1}\left(x^{*}\right)=\alpha d\left(p^{*}\right)$ and $\phi_{1}\left(x^{\prime}\right)=\alpha d\left(p_{1}\right)$, where $\alpha=\left(d\left(p_{2}\right)-s_{2}\right) / d\left(p_{2}\right)$ (recall that $s_{2} \leq d\left(p_{2}\right)$ and, hence, $\left.\left(d\left(p_{2}\right)-s_{2}\right)^{+}=d\left(p_{2}\right)-s_{2}\right)$. Hence,

$$
\gamma=\alpha p^{*} d\left(p^{*}\right)-c\left(\alpha d\left(p^{*}\right)\right)-\left[\alpha p_{1} d\left(p_{1}\right)-c\left(\alpha d\left(p^{*}\right)\right)\right] .
$$


Letting $m_{\alpha}(p)=\alpha p d(p)-c(\alpha d(p))$ for each $p \in\left[p^{*}, \bar{p}\right]$, we have that $p^{*}$ is the unique maximizer of $m_{\alpha}$ in $\left[p^{*}, \bar{p}\right]$. Indeed, for each $p \in(\hat{p}, \bar{p})$,

$$
\begin{aligned}
& m_{\alpha}^{\prime}(p)=\alpha\left(d(p)+\left[p-c^{\prime}(\alpha d(p))\right] d^{\prime}(p)\right), \\
& m_{\alpha}^{\prime \prime}(p)=\alpha\left(d^{\prime}(p)\left[2-\alpha c^{\prime \prime}(\alpha d(p)) d^{\prime}(p)\right]+d^{\prime \prime}(p)\left[p-c^{\prime}(\alpha d(p))\right]\right)
\end{aligned}
$$

and, recall,

$$
m^{\prime}(p)=d(p)+\left[p-c^{\prime}(d(p))\right] d^{\prime}(p) .
$$

Since $-d^{\prime}\left(p^{*}\right) c^{\prime}\left(\alpha d\left(p^{*}\right)\right)<-d^{\prime}\left(p^{*}\right) c^{\prime}\left(d\left(p^{*}\right)\right)$, we have that $m_{\alpha}^{\prime}\left(p^{*}\right)<\alpha m^{\prime}\left(p^{*}\right)=0$. Moreover, for each $p>\hat{p}, p>c^{\prime}(d(p))>c^{\prime}(\alpha d(p))$ implies that $m_{\alpha}^{\prime \prime}(p)<0$. Thus, $m_{\alpha}$ is decreasing in $\left[p^{*}, \bar{p}\right]$ and, thus, $p^{*}$ is the unique maximizer of $m_{\alpha}$ in $\left[p^{*}, \bar{p}\right]$. It then follows that $\gamma>0$.

The second case is when $p_{2}=p^{*}$. Then $\phi_{1}\left(x^{\prime}\right)=\alpha d\left(p_{1}\right)$ where $\alpha=\left(d\left(p_{2}\right)-\right.$ $\left.s_{2}\right) / d\left(p_{2}\right)$ and $\alpha d\left(p^{*}\right) \leq \phi_{1}\left(x^{*}\right) \leq d\left(p^{*}\right)$. Indeed, if $\phi_{1}\left(x^{*}\right)<d\left(p^{*}\right)$, then $\phi_{1}\left(x^{*}\right)+$ $\phi_{2}\left(x^{*}\right)=d\left(p^{*}\right)$. Hence, using $p_{2}=p^{*}$ and $\phi_{2}\left(x^{*}\right) \leq s_{2}, \phi_{1}\left(x^{*}\right) \geq d\left(p_{2}\right)-s_{2}=$ $\frac{d\left(p_{2}\right)-s_{2}}{d\left(p_{2}\right)} d\left(p^{*}\right)$. Thus, $\gamma \geq m_{\alpha}\left(p^{*}\right)-m_{\alpha}\left(p_{1}\right)>0$.

The final case is $p_{2}>p^{*}$. In this case, $\gamma=m\left(p^{*}\right)-u_{1}\left(p_{1}, d\left(p_{1}\right), p_{2}, s_{2}\right) \geq m\left(p^{*}\right)-$ $m\left(p_{1}\right)>0$. This completes the proof.

Lemma 8. For each $i \in\{1,2\}$ and $p_{i} \in\left(c^{\prime}(0), p^{*}\right),\left(p_{i}, \phi^{*}\left(p_{i}\right)\right)$ is not weakly dominated for $i$.

Proof. Let $i=1$ without loss of generality and $p_{1} \in\left(c^{\prime}(0), p^{*}\right)$. Suppose that there exists $\mu_{1} \in M_{1}$ that weakly dominates $\left(p_{1}, \phi^{*}\left(p_{1}\right)\right)$. Consider a sequence $\left\{p_{2, k}\right\}_{k=1}^{\infty}$ such that $p_{2, k} \downarrow p_{1}$ and $\mu_{1}\left(\left\{p_{2, k}\right\}\right)=0$ for each $k \in \mathbb{N}$; such sequence exists because the set of atoms of $\mu_{1}$ is countable. Let $s_{2, k}=d\left(p_{2, k}\right)$ for each $k \in \mathbb{N}$. Then, for each $k \in \mathbb{N}, u_{1}\left(p_{1}, \phi^{*}\left(p_{1}\right), p_{2, k}, s_{2, k}\right)=m\left(p_{1}\right), u_{1}\left(\mu_{1}, p_{2, k}, s_{2, k}\right) \leq \int_{\left[0, p_{2, k}\right)} m(p) \mathrm{d} \mu_{1}(p)$ (equality holds if $\mu_{1}\left(\left\{(p, s): s=\phi^{*}(p)\right\}\right)=1$ ) and

$$
\int_{\left[0, p_{2, k}\right)} m(p) \mathrm{d} \mu_{1}(p) \geq u_{1}\left(\mu_{1}, p_{2, k}, s_{2, k}\right) \geq u_{1}\left(p_{1}, \phi^{*}\left(p_{1}\right), p_{2, k}, s_{2, k}\right)=m\left(p_{1}\right) .
$$

Letting $f=m 1_{\left[0, p_{1}\right]}$ and, for each $k \in \mathbb{N}, f_{k}=m 1_{\left[0, p_{2, k}\right)}$, we have that $f_{k} \downarrow f$. Hence, by the monotone convergence theorem,

$$
\int_{\left[0, p_{1}\right]} m(p) \mathrm{d} \mu_{1}(p)=\lim _{k} \int_{\left[0, p_{2, k}\right)} m(p) \mathrm{d} \mu_{1}(p) \geq m\left(p_{1}\right) .
$$


By Lemma 2.3, we have that $m(p)<m\left(p_{1}\right)$ for each $p<p_{1}$. This, together with $\int_{\left[0, p_{1}\right]} m(p) \mathrm{d} \mu_{1}(p) \geq m\left(p_{1}\right)$ then implies that $\mu_{1}\left(\left\{p_{1}\right\} \times\left[0, d\left(p_{1}\right)\right]\right)=1$. But then $\mu_{1}$ does not weakly dominate $\left(p_{1}, \phi^{*}\left(p_{1}\right)\right)$. Indeed, this is clear if $\mu_{1}=\delta_{\left(p_{1}, \phi^{*}\left(p_{1}\right)\right)}$ since then $u_{1}\left(p_{1}, \phi^{*}\left(p_{1}\right), p_{2}, s_{2}\right)=u_{1}\left(\mu_{1}, p_{2}, s_{2}\right)$ for each $\left(p_{2}, s_{2}\right) \in X_{2} ;$ if $\mu_{1} \neq \delta_{\left(p_{1}, \phi^{*}\left(p_{1}\right)\right)}$, then $u_{1}\left(p_{1}, \phi^{*}\left(p_{1}\right), p_{2}, s_{2}\right) \geq u_{1}\left(\mu_{1}, p_{2}, s_{2}\right)$ for each $\left(p_{2}, s_{2}\right) \in X_{2}$ by Lemma 5 .

Combining Lemmas $5-8$, it follows that

$$
C_{i}=\left\{\left(p_{i}, s_{i}\right) \in X_{i}: p_{i} \in\left[c^{\prime}(0), p^{*}\right], s_{i}=\phi^{*}\left(p_{i}\right)\right\}
$$

We turn now to showing that $G_{B}$ is admissibly secure, which we will do by establishing the conditions in Remark 4.

Lemma 9. The game $G_{B}$ is admissibly reciprocal upper semicontinuous.

Proof. It follows by Aliprantis and Border (2006, Lemma 17.30, p. 569) that $u_{1}+u_{2}$ is upper semicontinuous. Thus, the conclusion follows by Carmona (2013, Theorem 3.20 , p. 39).

Lemma 10. The game $G_{B}$ is admissibly payoff secure.

Proof. We first note that it suffices to show that the following condition $(*)$ holds: For each $i \in\{1,2\}, \varepsilon>0$ and $\sigma \in M_{i} \times M_{j}^{0}$, there exists $\beta \in\left[c^{\prime}(0), p^{*}\right]$ such that $\sigma_{j}\left(\left\{\left(\beta, \phi^{*}(\beta)\right)\right\}\right)=0$ and $u_{i}\left(\beta, \phi^{*}(\beta), \sigma_{j}\right)>u_{i}(\sigma)-\varepsilon$. Indeed, suppose that condition (*) holds and that $G$ fails to be admissibly payoff secure. Then there is $i \in\{1,2\}$, $\varepsilon>0$ and $\sigma \in M_{i} \times M_{j}^{0}$ such that, for each $\mu_{i} \in M_{i}^{0}$ and open neighborhood $U$ of $\sigma$, $u_{i}\left(\mu_{i}, \sigma_{j}^{\prime}\right) \leq u_{i}(\sigma)-\varepsilon$ for some $\sigma^{\prime} \in U \cap M^{0}$. By condition $(*)$, let $\beta \in\left[c^{\prime}(0), p^{*}\right]$ such that $\sigma_{j}\left(\left\{\left(\beta, \phi^{*}(\beta)\right)\right\}\right)=0$ and $u_{i}\left(\beta, \phi^{*}(\beta), \sigma_{j}\right)>u_{i}(\sigma)-\varepsilon$. As $\delta_{\left(\beta, \phi^{*}(\beta)\right)} \in M_{i}^{0}$, for each $k \in \mathbb{N}$, pick $\sigma_{k} \in M^{0}$ such that $u_{i}\left(\beta, \phi^{*}(\beta), \sigma_{k, j}\right) \leq u_{i}(\sigma)-\varepsilon$ and $\sigma_{k, j} \rightarrow \sigma_{j}$. We have that $\tau_{\delta_{\left(\beta, \phi^{*}(\beta)\right)}, \sigma_{j}}\left(\left\{x \in X: u_{i}\right.\right.$ is discontinuous at $\left.\left.x\right\}\right)=\sigma_{j}\left(\left\{\left(\beta, \phi^{*}(\beta)\right)\right\}\right)=0$, and hence $u_{i}\left(\beta, \phi^{*}(\beta), \sigma_{k, j}\right) \rightarrow u_{i}\left(\beta, \phi^{*}(\beta), \sigma_{j}\right)$. Thus, we get $u_{i}(\sigma)-\varepsilon<u_{i}\left(\beta, \phi^{*}(\beta), \sigma_{j}\right) \leq$ $u_{i}(\sigma)-\varepsilon$, a contradiction. This contradiction shows that condition $(*)$ is sufficient for $G$ to be admissibly secure. 
In what follows we verify that condition $(*)$ hold. Let $i=1$ without loss of generality, $\varepsilon>0$ and $\sigma \in M_{1} \times M_{2}^{0}$. Let $A_{2}=\left\{p \in P: \sigma_{2}\left(\left\{\left(p, \phi^{*}(p)\right)\right\}\right)>0\right\}$ be the set of atoms of $\sigma_{2}$ and note that $A_{2}$ is countable.

If $u_{1}(\sigma) \leq 0$, pick $\beta \in\left[c^{\prime}(0), p^{*}\right] \backslash A_{2}$ and note that $u_{1}\left(\beta, \phi^{*}(\beta), \sigma_{2}\right) \geq 0$. Thus, we may assume that $u_{1}(\sigma)>0$.

There exists $\alpha \in P$ such that $u_{1}\left(\alpha, \phi^{*}(\alpha), \sigma_{2}\right) \geq u_{1}(\sigma)$ since $\sigma_{1}$ is a probability measure and using Lemma 5. As $\phi^{*}(\alpha)=0$ if $\alpha \leq c^{\prime}(0)$ by Lemma 2.2, it follows that $\alpha>c^{\prime}(0)$. Moreover, we may take $\alpha \leq p^{*}$. Indeed, if $\alpha>p^{*}$, it follows by Lemma 7 that $\alpha$ can be replaced with $p^{*}$.

Let $h:[0, \alpha]^{2} \rightarrow[0, d(0)]$ be defined by setting, for each $p_{1}, p_{2} \in[0, \alpha]$,

$$
h\left(p_{1}, p_{2}\right)=\min \left\{\frac{d\left(p_{2}\right)-\phi^{*}\left(p_{2}\right)}{d\left(p_{2}\right)} d\left(p_{1}\right), \phi^{*}\left(p_{1}\right)\right\} .
$$

We have that

$$
\left(p_{1}, p_{2}\right) \mapsto p_{1} h\left(p_{1}, p_{2}\right)-c\left(h\left(p_{1}, p_{2}\right)\right):[0, \alpha]^{2} \rightarrow \mathbb{R}
$$

and $m$ are uniformly continuous. Hence, let $\delta>0$ be such that $|p-\alpha|<\delta$ implies that

$$
\left|\left[p h\left(p, p_{2}\right)-c\left(h\left(p, p_{2}\right)\right)\right]-\left[\alpha h\left(\alpha, p_{2}\right)-c\left(h\left(\alpha, p_{2}\right)\right)\right]\right|<\varepsilon \text { for all } p_{2} \in[0, \alpha]
$$

and

$$
|m(p)-m(\alpha)|<\varepsilon
$$

We may take $\delta$ to be such that $\alpha-\delta>c^{\prime}(0)$.

Choose $\beta \in(\alpha-\delta, \alpha)$ such that $\beta \notin A_{2}$. Hence, $\beta \in\left[c^{\prime}(0), p^{*}\right] \backslash A_{2}$. Let $\gamma\left(p_{2}\right)=$ $u_{1}\left(\beta, \phi^{*}(\beta), p_{2}, \phi^{*}\left(p_{2}\right)\right)-u_{i}\left(\alpha, \phi^{*}(\alpha), p_{2}, s_{2}\right)$ for each $p_{2} \in\left[c^{\prime}(0), p^{*}\right]$. We consider four cases.

Case 1: $p_{2}<\beta$. We then have that $\left|\gamma\left(p_{2}\right)\right|=\mid\left[\beta h\left(\beta, p_{2}\right)-c\left(h\left(\beta, p_{2}\right)\right)\right]-\left[\alpha h\left(\alpha, p_{2}\right)-\right.$ $\left.c\left(h\left(\alpha, p_{2}\right)\right)\right] \mid<\varepsilon$ by $(2)$.

Case 2: $\beta<p_{2}<\alpha$. Then $u_{1}\left(\alpha, \phi^{*}(\alpha), p_{2}, \phi^{*}\left(p_{2}\right)\right)=\alpha h\left(\alpha, p_{2}\right)-c\left(h\left(\alpha, p_{2}\right)\right) \leq$ $\alpha \phi^{*}(\alpha)-c\left(\phi^{*}(\alpha)\right)<\beta \phi^{*}(\beta)-c\left(\phi^{*}(\beta)\right)+\varepsilon=u_{1}\left(\beta, \phi^{*}(\beta), p_{2}, \phi^{*}\left(p_{2}\right)\right)+\varepsilon$ by $(3)$.

Case 3: $p_{2}=\alpha$. Let $\phi_{i} \leq \phi^{*}(\alpha) \leq d(\alpha)$ be such that $u_{1}\left(\alpha, \phi^{*}(\alpha), \alpha, \phi^{*}(\alpha)\right)=$ $\alpha \phi_{i}-c\left(\phi_{i}\right)$. Then, exactly as in Case 2 above, we obtain that $\gamma\left(p_{2}\right)>-\varepsilon$. 
Case 4: $p_{2}>\alpha$. We then have that $\left|\gamma\left(p_{2}\right)\right|=|m(\beta)-m(\alpha)|<\varepsilon$ by (3).

It then follows that $u_{1}\left(\beta, \phi^{*}(\beta), \sigma_{2}\right)>u_{1}\left(\alpha, \phi^{*}(\alpha), \sigma_{2}\right)-\varepsilon$ since $\sigma_{2} \in M_{2}^{0}$ and $\left(\beta, \phi^{*}(\beta)\right) \notin A_{2}$. As $u_{1}\left(\alpha, \phi^{*}(\alpha), \sigma_{2}\right) \geq u_{1}(\sigma)$, it follows that $u_{1}\left(\beta, \phi^{*}(\beta), \sigma_{2}\right)>u_{1}(\sigma)-$ $\varepsilon$, establishing condition $(*)$. This completes the proof of the lemma.

Theorem 2 now follows by combining Lemmas 9 and 10, Remark 4 and Theorem 1.

Remark 11. Using a result in Carmona and Podczeck (2018), we now show that the set of limit admissible equilibria is independent of the choice of the allocation $\phi$ that determines players' payoff functions in (1), in the following sense. Let $\tilde{\phi}$ be a measurable selection of $\Phi$ such that the conclusion of Lemma 4 holds. Furthermore, let $u_{i}^{\tilde{\phi}}$ be defined by replacing $\phi$ with $\tilde{\phi}$ in $(1)$ for each $i=1,2$ and let $G_{B}^{\tilde{\phi}}=\left(X_{1}, X_{2}, u_{1}^{\tilde{\phi}}, u_{2}^{\tilde{\phi}}\right)$. Then the closure of the set of undominated strategies of player $i$ in $G_{B}^{\tilde{\phi}}$ is still $C_{i}$ for each $i=1,2$; this holds because the specific definition of $\phi$ was only used to establish Lemma 4. More importantly, $L\left(G_{B}^{\tilde{\phi}}\right)=L\left(G_{B}\right)$.

The see the above, let $\tilde{X}_{i}=\left\{\left(p_{i}, s_{i}\right) \in X_{i}: s_{i}=\phi^{*}\left(p_{i}\right)\right\}$ for each $i=1,2$, $\tilde{G}_{B}=\left(\tilde{X}_{1}, \tilde{X}_{2}, u_{1}, u_{2}\right)$ and $\tilde{G}_{B}^{\tilde{\phi}}=\left(\tilde{X}_{1}, \tilde{X}_{2}, u_{1}^{\tilde{\phi}}, u_{2}^{\tilde{\phi}}\right)$. Note that $\tilde{X}_{i} \subseteq X_{i}$ and, hence, $\tilde{M}_{i} \subseteq M_{i}$ for each $i=1,2$ and $\tilde{M} \subseteq M$. Carmona and Podczeck (2018, Section 5.1) have shown that $E\left(\tilde{G}_{B}\right)=E\left(\tilde{G}_{B}^{\tilde{\phi}}\right)$. For each $G \in\left\{G_{B}, G_{B}^{\tilde{\phi}}\right\}$, we clearly have that $E(G) \cap \tilde{M} \subseteq E(\tilde{G})$; furthermore, we have that $E(\tilde{G}) \subseteq E(G) \cap \tilde{M}$ due to Lemma $5{ }^{6}$ As $M^{0} \subseteq \tilde{M}$, we then obtain that $L\left(G_{B}^{\tilde{\phi}}\right)=E\left(G_{B}^{\tilde{\phi}}\right) \cap M^{0}=E\left(\tilde{G}_{B}^{\tilde{\phi}}\right) \cap M^{0}=$ $E\left(\tilde{G}_{B}\right) \cap M^{0}=E\left(G_{B}\right) \cap M^{0}=L\left(G_{B}\right)$.

${ }^{6}$ To see this, let $\sigma \in E(\tilde{G}), i \in\{1,2\}$ and $\sigma_{i}^{\prime} \in M_{i}$. We clearly have that $\sigma \in \tilde{M}$. Define $f_{i}: X_{i} \rightarrow \tilde{X}_{i}$ by setting, for each $\left(p_{i}, s_{i}\right) \in X_{i}, f\left(p_{i}, s_{i}\right)=\left(p_{i}, \phi^{*}\left(p_{i}\right)\right)$. Then, for each $\left(p_{i}, s_{i}, p_{j}, x_{j}\right) \in X, u_{i}\left(p_{i}, s_{i}, p_{j}, x_{j}\right) \leq u_{i}\left(f\left(p_{i}, s_{i}\right), p_{j}, x_{j}\right)$ by Lemma 5 . Hence, $u_{i}\left(\sigma_{i}^{\prime}, \sigma_{j}\right) \leq$ $\int_{X_{i}} u_{i}\left(f\left(p_{i}, s_{i}\right), \sigma_{j}\right) \mathrm{d} \sigma_{i}^{\prime}\left(p_{i}, s_{i}\right)=\int_{\tilde{X}_{i}} u_{i}\left(p_{i}, s_{i}, \sigma_{j}\right) \mathrm{d} \sigma_{i}^{\prime} \circ f^{-1}\left(p_{i}, s_{i}\right)=u_{i}\left(\sigma_{i}^{\prime} \circ f^{-1}, \sigma_{j}\right)$. As $\sigma_{i}^{\prime} \circ f^{-1} \in \tilde{M}_{i}$ and $\sigma \in E(\tilde{G})$, we have that $u_{i}\left(\sigma_{i}^{\prime} \circ f^{-1}, \sigma_{j}\right) \leq u_{i}(\sigma)$. Thus, $u_{i}\left(\sigma_{i}^{\prime}, \sigma_{j}\right) \leq u_{i}(\sigma)$ and, therefore, $\sigma \in E(G)$. 


\section{References}

Aliprantis, C., and K. Border (2006): Infinite Dimensional Analysis. Springer, Berlin, 3rd edn.

Andersson, O., C. Argenton, And J. Weibull (2014): "Robustness to Strategic Uncertainty," Games and Economic Behavior, 85, 272-288.

Bagh, A., And A. Jofre (2006): "Reciprocal Upper Semicontinuity and Better Reply Secure Games: A Comment," Econometrica, 74, 1715-1721.

Bich, P. (2016): "Strategic Uncertainty and Equilibrium Selection in Discontinuous Games," Paris School of Economics.

Billingsley, P. (1999): Convergence of Probability Measures. Wiley, New York, 2nd edn.

Carbonell-Nicolau, O. (2011a): "On the Existence of Pure-Strategy Perfect Equilibrium in Discontinuous Games," Games and Economic Behavior, 71, 23-48.

(2011b): "Perfect and Limit Admissible Perfect Equilibria in Discontinuous Games," Journal of Mathematical Economics, 47, 531-540.

Carmona, G. (2013): Existence and Stability of Nash Equilibrium. World Scientific, Singapore.

Carmona, G., and K. Podczeck (2018): "Invariance of the Equilibrium Set of Games with an Endogenous Sharing Rule," Journal of Economic Theory, 177, 133.

Dasgupta, P., And E. Maskin (1986): "The Existence of Equilibrium in Discontinuous Economic Games, II: Applications," Review of Economics Studies, 53, 27-41.

FudenberG, D., And D. Levine (1986): "Limit Games and Limit Equilibria," Journal of Economic Theory, 38, 261-279. 
Harris, C., M. StinchCombe, and W. Zame (2005): "Nearly Compact and Continuous Normal Form Games: Characterizations and Equilibrium Existence," Games and Economic Behavior, 50, 208-224.

JaCkSOn, M., And J. Swinkels (2005): "Existence of Equilibrium in Single and Double Private Value Auctions," Econometrica, 73, 93-139.

KohlberG, E., And J.-F. Mertens (1986): "On the Strategic Stability of Equilibria," Econometrica, 54, 1003-1037.

Maskin, E. (1986): "The Existence of Equilibrium with Price-Setting Firms," American Economic Review, 76, 382-386.

REny, P. (1999): "On the Existence of Pure and Mixed Strategy Equilibria in Discontinuous Games," Econometrica, 67, 1029-1056.

- (2016): "Nash Equilibrium in Discontinuous Games," Economic Theory, 61, $553-569$.

Selten, R. (1975): "Reexamination of the Perfectness Concept for Equilibrium Points in Extensive Games," International Journal of Game Theory, 4, 25-55.

Simon, L., And M. Stinchcombe (1995): "Equilibrium Refinements for Infinite Normal-Form Games," Econometrica, 63, 1421-1443.

VAn Damme, E. (1991): Perfection and Stability of Nash equilibrium. Springer Verlag, Berlin. 